\title{
L'approche dramatique comme support didactique en classe de FLE
}

\section{${ }^{*}$ Dr. Rana HARB}

\section{Introduction}

Depuis l'avènement de l'approche communicative, puis actionnelle, dans la didactique du Français Langue Etrangère, l'apprentissage des langues à travers les techniques dramatiques, acquiert de plus en plus d'importance. L'intégration des activités dramatiques dans les cursus universitaires est un chantier éducatif nouveau qui est au cœur des débats. La définition d'Anne Ubersfeld insiste sur le fait que le théâtre est "un échange de paroles reposant sur les mêmes lois que tout échange dans la vie. "» Le théâtre investit donc la faculté de s'exprimer qui est, ellemême, la visée principale du cours de FLE. Cela implique que le théâtre pourrait être à la base d'une nouvelle approche communicative et actionnelle qui vise l'apprentissage des langues.

L'année académique est une période favorable à l'introduction des activités théâtrales. Généralement, les amateurs de la production dramatique sont nombreux dans les classes. Cependant la tâche n'est pas facile vu les difficultés qui entravent la réalisation du projet. La présente étude abordera la problématique de l'intégration des activités théâtrales en classe de fr ançais langue étrangère à l'université. Cette intégration offre de nombreux avantages

Maître de conférences - Département de français

Faculté de Pédagogie - Université d'Alexandrie

${ }^{1}$ UBERSFELD, Anne, Les termes clés de l'analyse du théâtre, Le Seuil, Paris, 1996, p.26. 
mais suscite en même temps plusieurs interrogations. Comment insérer l'approche dramatique et l'adapter à la visée communicative et actionnelle d'un cours de FLE ? Comment parvenir à engager les apprenants dans un tel projet sans bousculer le cours de leurs études ? Comment gérer le temps et adapter les exigences du programme de base aux activités théâtrales ?

La première partie de cette recherche vise à analyser la compétence communicative et les composantes langagières tout en soulignant l'importance des différentes activités orales. La deuxième partie sera consacrée à la présentation des différents atouts des activités théâtrales ainsi que les différentes stratégies d'application.

Cependant, il ne suffit pas d'évoquer les apports de l'intégration des activités théâtrales, mais il s'agit aussi de pointer les problèmes rencontrés par l'enseignant lors de l'application de cette formation. Nous dresserons ainsi, un plan de travail en proposant une nouvelle voie de réflexion. Dans la troisième partie de ce travail nous soulignerons l'importance du rôle de l'enseignant, dans l'approche théâtrale, en tant qu'animateur de la formation et déclencheur de la parole. La dernière partie propose une bibliographie et une sitographie pour développer le sujet.

Le théâtre est un outil didactique d'une importance considérable. Grâce au large éventail d'activités investies, il contribue à l'enrichissement de l'enseignement de la langue. Charles Buls affirme :

"Ressentie comme un facteur de progrès personnel et comme une aide à la réalisation d'objectifs éducatifs fondamentaux, la formation théâtrale ne peut qu'entraîner 
intérêt et enthousiasme. La prolonger devient souhaitable, la transmettre est une nécessité. »²

La formation théâtrale exige de développer la compétence communicative en se basant surtout sur les activités orales. Qu'est ce que l'oral ? Qu'est ce que la compétence communicative? A quoi renvoient ces notions?

En 2001, le $\mathrm{CECRL}^{3}$ définit les trois volets liés à l'expression orale : écouter, prendre part à une conversation et s'exprimer oralement en continu . L'oral est ainsi défini comme étant une activité de réception et de production monologique et dialogique. Quant à la notion, compétence communicative, elle n'est simple qu'en apparence. Elle nécessite la mobilisation de différentes composantes. Nous pouvons la considérer comme l'habilité qu'acquiert l'apprenant durant ses études, à communiquer en français dans des contextes et des conditions variés, en utilisant des stratégies verbales et non verbales ${ }^{4}$ afin de maintenir le contact et gérer la communication dans une situation de communication précise. Nous pouvons donc affirmer que l'acquisition d'une langue dépend du degré de l'acquisition de la compétence de communication de la

\footnotetext{
${ }^{2}$ BULS, Charles, PENNEQUIN, Simone, Théâtre école. Deux passions accordées, La Montagne magique, Bruxelles, 1997, p.8.

${ }^{3}$ CECRL (Cadre européen commun de référence pour les langues) est un document publié par le Conseil de l'Europe en 2001, qui définit des niveaux de maîtrise d'une langue étrangère en fonction de savoir-faire dans différents domaines de compétence. Ces niveaux constituent désormais la référence dans le domaine de l'apprentissage et de l'enseignement des langues dans de nombreux pays. Il constitue une approche totalement nouvelle qui a pour but de repenser les objectifs et les méthodes d'enseignement des langues et, surtout, il fournit une base commune pour la conception de programmes, de diplômes et de certificats. En ce sens, il est susceptible de favoriser la mobilité éducative et professionnelle. La version électronique du CECRL est disponible à télécharger sur : http://www.coe.int/T/DG4/Linguistic/Source/Framework_FR.pdf

${ }^{4}$ La communication verbale est liée à la manière de structurer les idées à travers le choix des mots et leur sens. La communication non-verbale est liée au langage corporel : gestes, mimiques, intonation de la voix, etc.
} 
langue cible. Le développement de la compétence communicative pendant le cours de FLE s'effectue donc, essentiellement, à travers les interactions qui se présentent sous forme d'activités d'expression orale. Tel est le but de toute formation en langue étrangère.

L'éventail de ces activités orales est vaste. Nous pouvons présenter à titre d'exemple les exercices d'improvisation et le jeu de rôle. L'improvisation est un exercice qui entraîne les apprenants à "répondre de façon appropriée à une situation inattendue. ${ }^{5}$ Les situations d'improvisation sont nombreuses. Elles suscitent la spontanéité des apprenants et les incitent à utiliser leurs acquis antérieurs au niveau du vocabulaire et de la syntaxe. Corinne Houben affirme :

"L'improvisation est un jeu libérateur. Elle entraine aussi à porter un regard sur soi et sur l'autre dans une situation de communication. ${ }^{6}$

Le jeu de rôle est une autre activité interactive qui a pour objet de favoriser chez l'apprenant une pratique de l'expression orale. Il permet de développer chez l'apprenant la dimension créatrice. Il démarre des personnages et l'apprenant va agir et parler selon les caractéristiques de son personnage. Le jeu de rôle ne s'improvise pas et doit être précédé d'un exercice linguistique précis sur lequel il s'appuie.

Ces deux démarches, l'improvisation et le jeu de rôle sont proches par leurs objectifs. Ils sont interchangeables mais indispensables dans l'enseignement de l'oral.

\footnotetext{
${ }^{5}$ Cf. RESSOUCHES, Elodie, Quelle pratique théâtrale ? Franc-parler, 2009. http://www.francparler-oif.org/images/stories/fiches/theatre pratique.htm ${ }^{6}$ HOUBEN, Corinne, Le théâtre et les enseignants, La Montagne magique, Bruxelles, 2001, p.11
} 
Cependant, les questions suivantes se poseraient avec acuité. Pourquoi intégrer les activités théâtrales dans l'enseignement et ne pas se contenter de ces activités proposées dans les méthodes de l'apprentissage du FLE ? Pourquoi avoir recours à une approche dramatique au sein d'une classe de FLE ? Pour quelles visées ? Pour quels objectifs?

En effet, l'adaptation du texte dramatique permet l'intégration de diverses activités qui visent à développer l'interaction verbale et la réhabilitation de la fonction communicative. Le théâtre peut être considéré comme étant une approche pédagogique adéquate à l'enseignement du français au sein des classes du FLE. Dans ce sens, le théâtre n'est pas un module à suivre ou une méthode d'enseignement mais il est utilisé comme un outil complémentaire qui s'adapte aux besoins langagiers des apprenants. La pratique du théâtre soutient l'approche communicative et s'adapte facilement aux exigences de la formation initiale ainsi qu'au niveau des apprenants. Dans une activité théâtrale, l'apprenant prend une position centrale. Il a le privilège d'utiliser un langage authentique et de le mettre en œuvre dans des situations réelles ou fictives en classe. Adrien Payet, spécialiste de l'enseignement du français par le théâtre, affirme :

"En proposant une activité théâtrale, on créée des situations de communication - certes fictives - mais qui permettent un réel échange langagier et humain. Pendant la réalisation des activités, les apprenants sont en immersion totale dans le monde théâtral et francophone. Ils jouent sur scène, à travers leurs personnages, des situations réalistes ou imaginaires du monde extérieur. $~^{7}$

${ }^{7}$ PAYET, Adrien, Activités théâtral en classe de langue, CLE international, Paris, 2010, p.14. 
Les activités théâtrales sont l'accompagnateur idéal de l'apprentissage du français. L'approche dramatique ne se base pas uniquement sur la compétence communicative, mais elle a aussi l'avantage de travailler toutes les autres compétences indispensables à l'apprenant. Elle présente à l'enseignant un large éventail de pratiques pédagogiques écrites et orales.

L'apprenant devient plus impliqué par rapport à la langue cible. Peu à peu, il se trouve dans la nécessité de penser et de s'exprimer en utilisant une langue qui lui était jusque là étrangère. Ses capacités de mémorisation sont développées grâce aux multiples répétitions. Il parvient ainsi à surmonter les «blocs langagiers $»^{8}$, à gérer le stress et à acquérir une confiance en soi. Payet souligne :

"Prendre plaisir à s'exprimer pleinement en langue étrangère est une satisfaction qui à seule donne un sens à l'activité. Les apprenants devenant acteurs de leur propre apprentissage, s'investissent davantage dans le cours. Il en résulte naturellement une hausse de motivation et de dynamisme dans les activités d'oral. En ce sens, le théâtre est un formidable moteur de motivation pour la classe.»9

De plus, lors des répétitions, les apprenants sont entraînés à faire face aux regards des autres, de prendre la parole en public et à se concentrer. L'activité théâtrale serait donc pour l'apprenant un moyen de franchir les barrières de sa timidité. Il apprend à travailler ses gestes, ses déplacements et son regard et à exprimer ses émotions et ses sentiments. L'apprenant est ainsi entraîné à explorer tous les moyens inhabituels de la communication. Il se reconstruit alors, un système de règles langagières, culturelles et

\footnotetext{
${ }^{8}$ WEBER, Véronique, Théâtre et FLE: Un enseignement transversal, Rapport de stage, Université de Savoie, 2000. Version électronique :

http://nicomede.org/fle/memoirev.htm

${ }^{9}$ PAYET, Adrien, Activités théâtral en classe de langue, op.cit., p.13
} 
sociales qui commandent l'utilisation de sa parole et, qui lui fournit une approche pragmatique favorable aux échanges conversationnels dans les différentes situations de communication.

Un nouvel aspect linguistique s'ajoute à la compétence communicative qui résulte de ce genre d'activités. Le théâtre est effectivement un atout pour travailler la prononciation, l'articulation et les liaisons. Les exercices de phonétique aident l'apprenant à prendre conscience de sa voix. Il parvient ainsi à concevoir l'importance des pauses, de la ponctuation et pourra ensuite rythmer correctement les phrases. Les exercices répétés de phonétique aident les apprenants à prononcer les phonèmes difficiles. Ces exercices de diction, qui sont généralement jugés comme ennuyeux dans un programme figé, deviennent source de jeu. Les apprenants trouvent même un grand plaisir à répéter les scènes, sans lassitude, voire même avec patience et concentration, stimulés par leur désir de perfection. Véronique Weber affirme :

«Ce sont les ressources du jeu théâtral qui permettent à l'apprenant de corriger sa prosodie au sein d'un contexte facilitant. Ce contexte évite les effets démotivants d'une répétition systématique. Le fait de reprendre la prononciation en même temps que l'intention de jeu me paraît intéressante, car elle ôte en partie le caractère négatif d'une correction uniquement centrée sur la prosodie. En cherchant à améliorer le jeu du comédien en même temps que la langue, on gomme l'aspect parfois gênant de la "reprise" à froid. De plus, on sait qu'une correction à l'oral par la simple écoute, et la répétition de 
mots hors contexte est jugée inefficace par les didacticiens. ${ }^{10}$

Une nouvelle dimension sociale s'ajoute à la pratique du théâtre c'est l'acquisition de l'esprit du groupe. Les apprenants appréhendent les règles du travail en commun. Ils prennent conscience de la nécessité de s'entraider. L'engagement collectif est le seul moyen de l'avancement de l'activité théâtrale. Des stratégies socio-affectives s'installent au sein du groupe: coopération, entraide, soutien, contrôle des émotions, etc... L'apprenant découvre les codes sociaux de la culture française. Il parvient ainsi à développer ses capacités langagières. C'est là qu'intervient l'aspect socio-culturel de la compétence communicative.

$\mathrm{Au}$ début, le groupe s'organise. Chaque apprenant joue un double rôle ; il sera à la fois acteur et spectateur. D'une part, il participe à la présentation, en interprétant un personnage, et d'autre part il apprend à regarder, à critiquer et à s'auto-évaluer. Même les étudiants les plus timides parviennent à trouver leur place. Un esprit de coopération et de solidarité s'installe ainsi au sein du groupe. Le théâtre aide donc à "permettre une structuration individuelle dans un projet collectif. ${ }^{11}$

L'activité théâtrale est donc un engagement collectif qui permet à l'apprenant de trouver un rapport nouveau avec le langage tout en s'ouvrant sur les autres. Stéphanie Clerc souligne lors d'un atelier de théâtre :

«Cet atelier avait amélioré la cohésion du groupe par plus de mixité, par un engagement collectif plus soutenu et un plus grand désir de parole dans la langue de

${ }^{10}$ WEBER, Véronique, op.cit.

${ }^{11}$ WEBER, Véronique, Théâtre et FLE: Un enseignement transversal, op.cit. 
scolarisation. L'atelier semble avoir opéré comme un formidable générateur de motivation et un déclencheur des efforts nécessaires dans l'apprentissage d'une langue nouvelle. Les élèves plus avancés, démotivés en classe, se sont sentis valorisés par leur performance sur scène et par leur rôle d'experts auprès des plus faibles qu'ils soutenaient dans leurs efforts d'appropriation du texte et du jeu. ${ }^{12}$

Cependant, le jeu dramatique revêt une autre dimension importante. Un texte théâtral est toujours lié à un contexte idéologique et sociologique. Ainsi, littérature, civilisation et culture ne sont pas exclues du jeu. Au contraire, le théâtre a le privilège de ranimer les différentes périodes historiques et littéraires. La vision de la pièce est explicitement présentée et l'apprenant se trouve ainsi face à un contexte socio-culturel différent du sien.

La production langagière de l'apprenant se trouve ainsi liée à l'activité sociale. Le théâtre a l'avantage de sensibiliser l'apprenant à la culture française tout en lui gardant son autonomie. Il entre ainsi en interaction directe avec la culture française, qui s'avère indiscutablement indissociable de l'apprentissage de toutes les langues étrangères. Wei Chen souligne :

"En ce sens, l'apprenant de français est censé non seulement de se familiariser avec la culture véhiculée par la langue française, mais encore dépasser ses propres conditionnements et stéréotypes culturels pour se rendre capable de s'exprimer aussi spontanément qu'un français natif et d'être accepté et compris sans difficulté par ce dernier. C'est-à-dire qu'il doit surmonter les obstacles interculturels, qui peuvent intervenir lors de la communication et qui risquent de brouiller cette dernière.

${ }^{12}$ CLERC, Stéphanie, Apports de la pratique théâtrale en classe d'accueilhttp://www.francparleroif.org/images/stories/dossiers/clerc_2009.htm 
Sur ce plan, le théâtre en langue étrangère pourrait jouer un rôle efficace. ${ }^{13}$

Nous pouvons dire que toutes les composantes de l'acte de parole sont ainsi implicitement investies dans les activités théâtrales. ${ }^{14}$ Associer l'apprentissage du français à des activités théâtrales est une démarche pédagogique innovante et ludique à la fois. Cette pratique, associée à une méthode de l'enseignement du français langue étrangère, répond aux objectifs et aux normes du CECRL. Elle tend à promouvoir l'accès direct à la parole tout en s'amusant.

Il s'agit, maintenant, de trouver les pistes favorables pour préparer un projet théâtral en classe. Plusieurs types de projets peuvent être proposés aux apprenants. Un projet à court terme consiste à préparer une fable ou plus simplement assister à une pièce de théâtre. Cependant, un projet à long terme tel que l'élaboration d'un spectacle, nécessite un travail laborieux et interdisciplinaire.

Nous tenterons dans cette partie de ce travail de dresser un plan des démarches à entreprendre lors de l'élaboration d'un tel projet. Le dossier conçu et réalisé par

\footnotetext{
${ }^{13}$ WEI, Chen, « Un regard nouveau sur le théâtre en tant que support pédagogique du FLE », Universités des études internationales de Shanghai in Synergies Chine, n.4, 2009, p.42.

${ }^{14}$ Les composantes psychologiques mettent en jeu l'image de soi en mobilisant les ressources linguistiques. Les composantes physiques mobilise l'appareil phonatoire (respiration, voix, intonation) ainsi que le corps (mimique, regard). Les composantes pragmatiques sont celles qui renvoient à comprendre la situation de communication et adapter le langage. Les composantes discursives renvoient à la maîtrise des conduites discursives (raconter, expliquer, argumenter). Les composantes linguistiques renvoient aux règles de fonctionnement de grammaire, du lexique, etc. Les composantes prosodiques renvoient à l'intensité du ton attribuée à la parole. Les composantes métalinguistiques renvoient à la capacité à contrôler, à clarifier et à reformuler le discours. La composante interactive renvoie à l'oral de l'interaction de la conversation face à face.
} 
Emeline Giguet, Haydée Maga et Elodie Ressouchesd propose un plan des démarches à suivre. ${ }^{15}$

L'enseignant commencera d'abord par former une équipe aux compétences variées. Tous les apprenants seront invités à participer. La première étape doit être centrée sur le choix du texte qui sera travaillé. Cette étape est importante et peut être investie par l'enseignant afin de développer les compétences de compréhension écrite chez les apprenants.

Le formateur commencera par introduire les œuvres d'une manière académique (étude du lexique, des courants littéraires, du style de l'auteur, etc.). L'apprenant est censé par la suite faire un résumé de la pièce ou rédiger un compte rendu du cours théorique. L'expression écrite est ainsi investie. ${ }^{16}$

Le recours aux supports audio-visuels pour faire voir et écouter aux apprenants des extraits de pièces de théâtre, est indispensable. Cela permettra à l'enseignant de travailler la compréhension orale. Ces supports sonores aideront de même les apprenants à perfectionner leur diction, leur intonation et leur prononciation.

Le texte, ensuite, doit être mémorisé. Ce travail pourrait être long et difficile pour certains apprenants. Le formateur aura recours à l'autodictée qui aidera sans doute les apprenants les plus faibles. Les apprenants doivent être livrés ensuite à des exercices de récitation du texte, jusqu'à perfection, avant d'entamer la mise en scène individuelle puis collective.

${ }^{15}$ Cf., GIGUET, Emeline, MAGA, Haydée, RESSOUCHES, Elodie, Enseigner le français avec le théâtre, Franc-parler http://www.francparleroif.org/images/stories/dossiers/theatre.htm

${ }^{16}$ Le professeur peut initier ses étudiants à la rédaction de saynètes, ou de sketches selon leur niveau. 
Le formateur devrait aussi s'attarder aux didascalies. Un groupe d'apprenants sera chargé de dresser une liste de tout ce qui concerne les costumes, les décors, les accessoires, les installations techniques de lumière et sons. La gestion du temps est d'une grande importance si l'enseignant envisage de présenter la pièce dans un spectacle public.

Au fur et à mesure de l'élaboration des activités, la classe se transforme en un milieu social. Chaque apprenant a une tâche à accomplir. Ce qui permet à l'enseignant d'orienter les activités des apprenants, fixer les objectifs, définir les obstacles et mobiliser toutes les ressources langagières des apprenants pour les surmonter. Ces activités théâtrales permettent d'acquérir non seulement des savoirfaire mais aussi des savoir-être et des savoir-apprendre, tout en focalisant l'apprentissage sur la langue. De là, nous accédons à une nouvel approche qui est l'approche actionnelle. $^{17}$

L'objectif essentiel de l'intégration des pratiques théâtrales dans l'enseignement du FLE, serait alors le déblocage, c'est-à-dire parvenir à libérer la parole des apprenants et de faire des mots et des règles de la syntaxe et de la grammaire un moyen et non une visée en soi.

Tout au long du parcours, plusieurs difficultés pourraient entraver la progression de la formation.

\footnotetext{
${ }^{17}$ La perspective actionnelle est un des piliers du CECRL. Elle s'inspire de l'approche communicative tout en y ajoutant le concept de l'accomplissement de tâche. L'apprenant, confronté à des situations de la vie sociale, se trouverait dans la nécessité de mobiliser ses compétences et ses ressources langagières afin de réussir sa communication. L'apprenant devient non seulement un apprenant-usager de la langue, mais il devient aussi un acteur social.

Cf. GIRARDET, Jacky, «Enseigner le FLE selon une approche actionnelle : Quelques propositions méthodologiques », ACTES DU XIIème COLLOQUE PÉDAGOGIQUE DE L'ALLIANCE FRANÇAISE DE SÃO PAULO, CLE International, 2011, p.3-4 Cf. TAGLIANTE, Christine, L'évaluation et le cadre européen commun, CLE international, Paris, 2005, p.36.
} 
Les étudiants, livrés à eux même pendant les répétitions, ne suivront généralement pas les exigences et le sérieux des exercices. L'assistance permanente et la disponibilité de l'enseignant serait donc indispensable.

Certains apprenants pourraient être peu intéressés et se sentiraient obligés de suivre la formation. Ils risqueraient d'avoir une attitude passive. L'enseignant devrait alors attirer leur attention, travailler sur l'intéressement et changer de méthodologie selon le cas.

De même, les apprenants n'ont pas tous le même niveau de compétences langagières. Certains monopolisent la parole tandis que d'autres se contentent d'écouter l'enseignant. Ces derniers ont généralement peur du ridicule et du jugement des autres apprenants. L'enseignant devrait les valoriser et éviter toutes réprimandes.

Parfois, la disposition d'une salle de présentation s'avère problématique. Cependant, il est important de fixer un lieu de travail, un espace scénique, une scène de présentation. L'enseignant pourrait alors adapter le lieu disponible afin de créer un espace scénique convenable.

Les problèmes financiers constituent l'obstacle majeur pour toutes les activités universitaires. Généralement, les établissements ne possèdent pas de ressources financières suffisantes pour supporter les projets théâtraux. L'enseignant doit savoir se dépanner. Il est indispensable qu'il ait recours à l'imagination et à la création et utiliser tous les moyens disponibles pour les costumes et les décors: vêtements, tissus, carton, maquillage, masques, etc. Toutefois, l'étape de planification est propice et permettra d'identifier les obstacles, parvenir à les surmonter et garantir le succès de la formation.

Une situation d'apprentissage comporte trois pôles: le formateur, l'apprenant et le contenu. L'efficacité de l'enseignement dépend de la réalisation du processus 
d'animation avec les apprenants. L'enseignant doit parvenir à connaître les besoins de ses apprenants, à fixer ses objectifs et à adapter les actions d'apprentissage.

Le formateur joue ainsi un rôle primordial dans la préparation des activités théâtrales. Son rôle ne se limite pas à assurer la continuité des séances ou l'organisation des activités. Ses attitudes envers ses apprenants ainsi que ses compétences déterminent la qualité de la formation. Les stratégies d'aide et de guidage sont précisées, nous nous baserons sur la notion d'étayage développé par Bruner en 1983. Il explique :

«La plupart du temps, l'intervention d'un tuteur comprend une sorte de processus d'étayage qui rend l'enfant ou le novice capable de résoudre un problème, de mener à bien une tâche ou d'atteindre un but qui auraient été, sans cette assistance, au-delà de ses possibilités. ${ }^{18}$

Selon Bruner, l'étayage est le soutien pédagogique que peut apporter l'adulte à une personne moins experte afin de lui permettre d'accomplir une tâche. Cette aide pourrait avoir la forme d'un contrat didactique. ${ }^{19}$ Guy Brousseau a introduit le concept de ce contrat didactique en 1978 : c'est

${ }^{18}$ Bruner, Jérôme Seymour, Le développement de l'enfant : savoir faire, savoir dire, PUF, Paris, 1983. P.263.

${ }^{19}$ Un contrat didactique est un contrat qui est établi entre le professeur et les élèves en relation avec le savoir. Ce contrat fixe les rôles, places et fonctions de chaque partie. Il fixe de même, les activités du professeur et des élèves, les places respectives de chacun par rapport au savoir inculqué et même les conditions générales dans lesquelles ces rapports au savoir évolueront au cours de l'enseignement. Le contrat didactique agit comme un facteur d'équilibre entre professeur, savoir et élève. Par contre le contrat pédagogique est constitué de l'ensemble des règles de vie à suivre dans une classe. La nature de ce contrat n'est

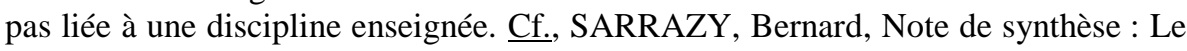
contrat didactique in Revue française de pédagogie, volume 112, Didactique des sciences économiques et sociales, 1995, pp. 85-118. 
" une relation qui détermine, explicitement pour une petite partie, mais surtout implicitement ce que chaque partenaire, l'enseignant et l'enseigné, a la responsabilité de gérer et dont il sera responsable d'une manière ou d'une autre devant l'autre. Ce système d'obligation réciproque ressemble à un contrat. ${ }^{20}$

Au cours de l'activité théâtrale , l'enseignant doit avoir recours non seulement au langage mais aussi aux procédés non-verbaux de la communication telle que la gestuelle. Effectivement, langage et gestes sont indissociables dans l'apprentissage de la langue. Les gestes, les mimiques et la voix ont un fort impact sur la compréhension et la mémorisation. La langue "ne se présente jamais nue mais au contraire toujours habillée du costume de la voix du locuteur et du pardessus de ses attitudes, gestes, mimiques et regards. ${ }^{21}$

De même, une des plus importantes exigences du formateur, et qui est en rapport direct avec le volet socio-culturel de la compétence de communication, est la gestion du groupe et surtout la gestion des conflits au sein du groupe. Un atelier de théâtre est un jeu coopératif où l'enseignant joue le rôle de l'animateur : il définit la durée et l'espace de l'activité, distribue des tâches précises aux apprenants, indique les modalités du travail, intervient lors des blocages et contrôle la réticence des plus timides en les incitant à participer à ce projet collectif.

Toutefois et malgré toutes ses obligations, au terme $\mathrm{du}$ projet, l'enseignant ressentira une grande satisfaction personnelle et professionnelle. Intégrant le ludisme au sein

${ }^{20}$ BROUSSEAU, Guy, Fondements et méthodes de la didactique des mathématiques. Recherche en didactique des mathématiques, La Pensée Sauvage, Grenoble, 1986, p.322-323.

${ }^{21}$ COLLETA, Jean-Marc, Communication non verbale et parole multimodale : quelles implications didactiques? in Le français dans le monde. Numéro spécial, Recherches et applications, 2005, p.1. 
de la formation, l'apprentissage de la langue revêt un intérêt nouveau. La pratique du théâtre se caractérise donc par sa globalité. Nous partageons l'avis de Jean-Claude Gal :

"La langue théâtrale me sert à tout instant. Elle est tour à tour, source de découvre, de saveur, de transmission. Au bout de quelques jours de travail et d'échange, elle devient complice d'une jouissance, qu'il s'agisse dans le cadre d'une joute oratoire, d'un affrontement ou d'une communion verbale. ${ }^{22}$

Malgré l'effort déployé, le climat du travail sera ludique et agréable. L'animateur saura investir l'ambition et l'énergie de ses étudiants. Dans un univers différent, les jeunes apprendront à jongler avec les mots, s'exprimer à travers la parole, la voix et le geste. Tâtonnant au début, le pas sûr ensuite, ils découvriront une nouvelle conception du savoir basée sur l'engagement personnel et le respect du groupe. 


\section{Conclusion}

Le théâtre a toujours tenu une place privilégiée parmi les disciplines enseignées à l'université mais il figure généralement comme un cours de théories appliquées sur des textes dramatiques ou sur une pièce de théâtre. Dans le cadre de l'enseignement d'une langue l'approche théâtrale constitue un outil didactique et pédagogique, à la fois, riche en ressources et motivant.

Ce que nous proposons ici, est de faire de ce module une discipline à part, située à mi-chemin entre apprentissage et ludisme. En tant que complémentaire à une méthode FLE, un tel projet aurait l'avantage de développer les compétences écrites et orales de l'apprenant. Les compétences communicatives et actionnelles seraient aussi exploitées. La démarche proposée dans ce travail stimulera l'apprenant et lui permettra de développer, non seulement ses capacités d'expression mais aussi sa manière d'appréhender la langue sous ses différentes formes. L'approche théâtrale ajoute à l'apprentissage une dimension corporelle, vocale, émotionnelle, artistique et culturelle.

Elle est la voie "pour alimenter l'imagination, nourrir l'intelligence: le théâtre peut être un fabuleux déclencheur d'étincelles. Pluriel et infini, le théâtre relève aussi de l'expérience vécue, celle de l'expression. $»^{23}$

L'approche dramatique est une voie didactique idéale pour lier la pédagogie en classe à la création et à l'art.

${ }^{23}$ DELDIME, Roger et Coll., L'éducation des jeunes au théâtre, op.cit., p.9. 
L'expérience théâtrale est un lieu d'épanouissement individuel et une expérience qui donne à penser et à s'exprimer. Christiane Page affirme :

"Cela implique que sa parole et sa pensée soient dignes d'intérêt avant qu'il ne soit adulte et qu'il ait officiellement le droit de penser pour rejoindre ceux qui monopolise le pouvoir que confère la faculté de $s^{\prime}$ exprimer. ${ }^{24}$

Il nous reste à lancer un appel aux établissements académiques de s'ouvrir au développement de l'approche dramatique et de la considérer comme une des didactiques les plus fécondes dans l'apprentissage du français. Associant le milieu éducatif à la création artistique, l'enseignement de la langue revêt une vision nouvelle. Nos étudiants ne méritent-ils pas une telle expérience, une telle chance d'être à la fois chercheur, joueur, acteur, penseur et détenteur de la parole?

\footnotetext{
${ }^{24}$ PAGE, Christiane, «Le jeu dramatique : une rencontre entre Théâtre et éducation », Apprendre par le théâtre in Revue Etudes Théâtrales, de l'UCL, n.34, 2005.
} 
Bibliographie

1. AGULAR, Claudio, « Qu'est-ce-que le jeu de rôle ? » in Le Français dans le monde, n.176, 1983.

2. BESSON, Jean-Louis, "Apprendre par le théâtre » in Revues études théâtrales de l'UCL, n.34, 2005.

3. BROUSSEAU, Guy, Fondements et méthodes de la didactique des mathématiques. Recherche en didactique des mathématiques, La Pensée Sauvage, Grenoble, 1986.

4. Bruner, Jérôme Seymour, Le développement de l'enfant : savoir faire, savoir dire, PUF, Paris, 1983.

5. BULS, Charles, PENNEQUIN, Simone, Théâtre école: Deux passions accordées, La Montagne magique, Bruxelles, 1997.

6. CLERC, Stéphanie, Apports de la pratique théâtral en classe d'accueil, version électronique : http://www.francparleroif.org/images/stories/dossiers/ clerc 2009.htm

7. CHISS, Jean-Louis, DAVID Jacques, REUTER, Yves, Didactique du français: fondement d'une discipline, Editions de Boeck Université, Belgique, 2008.

8. COLLETA, Jean-Marc, «Communication non verbale et parole multimodale : quelles implications didactiques ?» in Le français dans le monde. Numéro spécial, Recherches et applications, 2005.

9. DESMARETS, Michel, Enseigner le théâtre aux adolescents : un métier paradoxal, version électronique:

http://www.bellone.be/docs/bonus23a.pdf 
10.DELDIME, Roger et Coll., L'éducation des jeunes au théâtre, La Montagne magique, Bruxelles, 1998.

11.DELDIME, Roger, Fou de théâtre Lansman, Belgique, 1993.

12.DELDIME, Roger et Coll., Le théâtre et les enseignants, La Montagne magique, Bruxelles, 2001.

13.FLORENCE, Jean, «Une scène pour l'adolescent: l'école du jeu » in Actes du colloque de l'UCL, Apprendre par le théâtre, n.34, 2004.

14.GAL, Jean-Claude, Un théâtre \& des adolescents, Service universités culture, Théâtre du Pélican, Presses Universitaires Blaise Pascal, Paris, 2006.

15.GIGUET, Emeline, MAGA,Haydée, RESSOUCHES, Elodie, Enseigner le français avec le théâtre, Francparler version électronique http://www.francparleroif.org/images/stories/dossiers/theatre.htm

16.GIRARDET, Jacky, "Enseigner le FLE selon une approche actionnelle: Quelques propositions méthodologiques » in Actes du XIIème Colloque pédagogique de l'Alliance française de São Paulo, CLE International, 2011.

17.HOUBEN, Corinne, Le théâtre et les enseignants, La Montagne magique, Bruxelles, 2001.

18.LAURENS, Véronique, Théâtre et apprentissage du français, La Cimade, Paris, 2005.

19.PAGE, Christiane, «Le jeu dramatique: une rencontre entre Théâtre et éducation », Apprendre par le théâtre in Revue Etudes Théâtrales, de 1'UCL, n.34, 2005.

20.PAYET, Adrien, Activités théâtral en classe de langue, CLE international, Paris, 2010.RESSOUCHES, Elodie, Quelle pratique théâtrale? Franc-parler, 2009, version électronique http://www.francparleroif.org/images/stories/fiches/theatre_pratique.htm

$$
0 .
$$


21.SARRAZY, Bernard, Note de synthèse : Le contrat didactique in Revue française de pédagogie, volume 112, Didactique des sciences économiques et sociales, 1995.

22.SPILMANN, Guy, « La compétence communicative : Les situations d'utilisation », in BLA Les essentiels apprendre et enseigner le FLE, octobre 2013, version électronique :http://faculty.georgetown.edu/spielmag/ docs/FLE/competence.htm

23.WEBER, Véronique, Théâtre et FLE: Un enseignement transversal, Rapport de stage, Université de Savoie, 2000. Version électronique : http://nicomede.org/fle/memoirev.htm

24.WEI, Chen, «Un regard nouveau sur le théâtre en tant que support pédagogique du FLE », Universités des études internationales de Shanghai in Synergies Chine, n.4, 2009.

\section{Sites internet :}

- Comédiathèque.net

- Dramaction

- Franc-parler.org

- Lepointdufle.net

- Le proscenium

- Remue.net

- Théâtre et FLE

- Théâtre en scène sur le site de TV5 


\section{Table des matières}

Introduction.........................................2

Compétences du langage.................................4

Intégration du théâtre dans la classe de FLE.............6

Etapes de la formation.............................12

Difficultés rencontrées................................14

Rôle de l'enseignant.................................15

Conclusion............................................ 19

Bibliographie.......................................21 


\section{L'approche dramatique comme support}

\section{didactique en classe de FLE}

Résumé :

Depuis l'avènement de l'approche communicative puis actionnelle dans la didactique du Français Langue Etrangère, l'apprentissage des langues à travers les techniques dramatiques acquiert de plus en plus d'importance. Cela implique que le théâtre pourrait être à la base d'une nouvelle approche communicative de l'apprentissage des langues. La présente étude abordera la problématique de l'intégration d'activités théâtrales en classe de français langue étrangère à l'université. Après avoir brièvement analysé la compétence communicative et les composantes langagières, dans la première partie de ce travail, nous présenterons les différentes activités orales. Dans la deuxième partie de ce travail, nous présenterons les différents atouts des activités théâtrales ainsi que les différentes stratégies d'application.

Cependant, il ne suffit pas d'évoquer les bienfaits de l'intégration des activités théâtrales, mais il s'agit de pointer les problèmes rencontrés par l'enseignant lors de l'application de cette formation. Nous dresserons ainsi, un plan de travail. Dans la troisième partie de ce travail nous soulignerons l'importance du rôle de l'enseignant en tant qu'animateur de la formation et déclencheur de la parole. La dernière partie vous propose une bibliographie et une sitographie. 


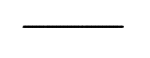

ملخص

عن طريق التواصل يتمكن الفرد من التفاعل مع افراد المجتع وتتميه

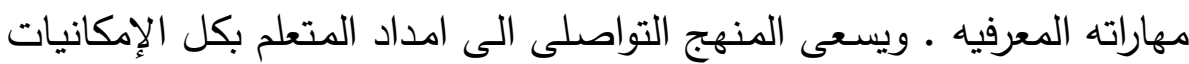

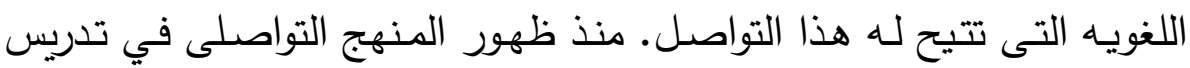
اللغه الفرنسيه كلغه أجنبيه أصبحت الدراما من التقنيات التى ازدادت أهميتها فى الآونه الأخيره. وبذلك يصبح المسرح وأنشطته المختلفه الشفهيه والتحريريـا من الأسس التى يقوم عليها المنهج التواصلى. تتناول هذه الدراسه أهميه تدريس الدراما كمنهج مكمل ومصاحب لتدريس اللغه الفرنسيه كلغه أجنبيه. بعد تحليل

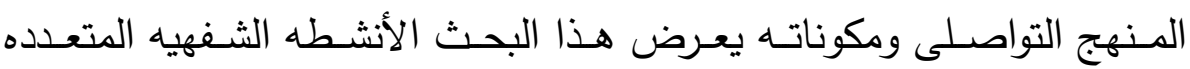
وأهميتها والية تنفيذها وإعتمادها كوسيله أساسيه فى تدريس اللغه الفرنسيه كلغه

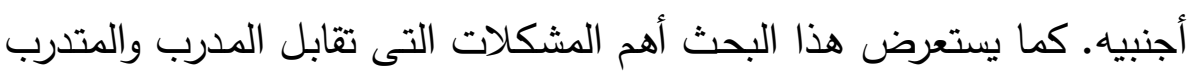
وسبل حلها. 